\title{
THE EFFECT OF L-CARNOSINE ON ERYTHROCYTE DEFORMABILITY AND AGGREGATION ACCORDING TO THE CELL AGE IN YOUNG AND AGED RATS
}

\author{
Gülten Erken', Melek Bor-Kucukatay², Emine KilicToprak², Beyza Akdag ${ }^{3}$, Vural Kucukatay
}

Balikesir University, Faculty of Medicine, Department of Physiology, Balikesir, Turkey ${ }^{1}$; Pamukkale University, Faculty of Medicine, Department of Physiology, Kinikli, Denizli, Turkey²; Pamukkale University, Faculty of Medicine, Department of Biostatistics, Kinikli, Denizli, Turkey ${ }^{3}$

\begin{abstract}
Summary: This study aimed to investigate alterations in hemorheology induced by L-carnosine, an anti- oxidant dipeptide, and to determine their relationship to oxidative stress in density-separated erythrocytes of aged and young rats. 28 male Sprague Dawley rats were divided into 4 groups as aged (Aca), young (Yca) L-carnosine groups (250 mg/kg L-carnosine, i.p.) and aged (As), young (Ys) control groups (saline, i.p.). Density separation was further performed to these groups in order to separate erythrocytes according to their age. Blood samples were used for the determination of erythrocyte deformability, aggregation; and oxidative stress parameters. Erythrocyte deformability of Yca group measured at $0.53 \mathrm{~Pa}$ was lower than Aca group. Similarly, deformability of least-dense (young) erythrocytes of Yca group was decreased compared to least-dense erythrocytes of Aca groups. Total antioxidant capacity (TAC) of Aca group was higher and oxidative stress index (OSI) lower than As group. Although L-carnosine resulted in an enhancement in TAC of aged rats, this favorable effect was not observed in erythrocyte deformability and aggregation in the dose applied in this study.
\end{abstract}

Key words: Aged rat; L-carnosine; Density separation; Erythrocyte deformability; Total antioxidant status; Total oxidant status

\section{Introduction}

Aging is characterized by macromolecular damage caused by free oxygen radicals and accumulation of oxidized/cross linked/denatured proteins (12). L-carnosine (Beta-alanyl-L-Histidine) is an endogenous dipeptide which is known to have antioxidant activity (8). It has been suggested that L-carnosine delays effects of aging, in relation to antioxidant activity, by preventing the formation of cross-linked, oxidated and denaturated proteins (13).

Erythrocytes are essential cells for tissue oxygenation. The ability of the entire red blood cell (RBC) to deform is of crucial importance for performing its function of oxygen delivery and it is also a determinant of the cell survival time in the circulation $(17,24)$. The physiological importance of erythrocyte aggregation in circulation is its tendency to increase the blood viscosity in low shear flow and to disturb the passage in capillary circulation through the formation of sludge $(3,22)$. RBC deformability is the ability of the cell to change its shape when passing through microcirculation or subjected to shear stress and it is an important determinant of blood flow particularly in microcirculation (23). Impaired RBC deformability in cellular aging has been previously reported (27). Oxidant stress is involved in mechanisms of biological aging as well as aging of the erythrocyte $(12,22)$.

$\mathrm{RBC}$ do not have protein synthesis mechanisms, and therefore the activity of most proteins diminishes throughout the life-span of these cells. Thus, target mechanisms of carnosine may be expected to be affected by cellular aging. Therefore, it might be suggested that the role of carnosine in modulating RBC mechanical properties may differ between $\mathrm{RBC}$ of different in vivo ages. In vitro L-carnosine was previously demonstrated to improve $\mathrm{RBC}$ deformability which was impaired by $\mathrm{H}_{2} \mathrm{O}_{2}$ treatment. Increase in $\mathrm{RBC}$ deformability was more prominent in young rats compared to elder ones (1). Although the effects of in vitro L-carnosine on RBC deformability was shown; as far as we know, there is no study in the literature demonstrating the effects of in vivo L-carnosine treatment on aggregation and deformability of density-separated erythrocytes in young and aged rats. Therefore, the aim of the present study was to investigate the effects of in vivo L-carnosine treatment on deformability and aggregation of young and aged erythrocytes of young and aged rats. Parameters indicating oxidative stress (total oxidant status, TOS; total antioxidant capacity, TAC and oxidative stress index, OSI) were also determined in order to investigate the relationship of oxidative stress to the possible alterations in hemorheological parameters. 


\section{Materials and methods}

\section{Animals and experimental procedure}

A total of twenty eight male Sprague Dawley young (3-4 months, 150-200 g) and aged (20-24 months, 300-340 g) rats were used for the experiments. The rats were fed with standard diet and water ad libitum. Animal handling during all experimental protocol was consistent with the National Institutes of Health Guidelines for the Care and Use of Laboratory Animals (NIH Publication No. 85-23) and approved by the Pamukkale University Ethics Committee of Animal Care and Usage. The animals were divided into four main groups: Aged L-carnosine (Aca), young L-carnosine (Yca) and aged control (As), young (Ys) control groups. Each group consisted of seven animals. A preliminary study was performed in order to determine the most effective dose and duration of L-carnosine administration. According to the results of the preliminary study, a single dose of L-carnosine (250 mg/kg; i.p.; Sigma, U.S.A.) was injected to the rats of the L-carnosine groups, while saline was injected to control groups. 30 minutes after the injection, the rats were anaesthetized with xylazine $(10 \mathrm{mg} / \mathrm{kg}$, i.p.) and ketamine $(90 \mathrm{mg} / \mathrm{kg}$, i.p.). Heparinized blood was collected from their abdominal aorta under anesthesia and used for the determination of hemorheological parameters. The reason why it is preferred to take the blood this way is that it allows maximum blood to be taken while the respiration of the animal continues. Therefore at least 8 milliliter of blood could be collected from each animal. The animals were then euthanased by exsanguination while under anesthesia. Plasma samples were stored at $-80^{\circ} \mathrm{C}$ until being used for the measurement of parameters representing total oxidant-antioxidant status.

\section{Density Separation of Erythrocytes}

Erythrocytes were separated, according to their density, using discontinuous Iodixanol (Optiprep, Nycomed Pharma As, Norway) density gradients (2). Five Iodixanol layers with densities between 1.075, 1.085, 1.095, 1.105, and $1.115 \mathrm{~g} / \mathrm{ml}(2 \mathrm{ml}$ each) were layered on top of each other in a test tube with the densest layer at the bottom. One milliliter of whole blood was carefully layered on top of the least dense layer, and the tube was centrifuged at $2,500 \mathrm{~g}$ for 25 min at $22{ }^{\circ} \mathrm{C}$. RBC were then harvested from specific density fractions: least dense cells were those that accumulated in $1.095 \mathrm{~g} / \mathrm{ml}$ layer and most dense cells were those that accumulated in the $1.105 \mathrm{~g} / \mathrm{ml}$ layer. RBC deformability was assessed for cells in these fractions as well as non-fractionated RBC (21). Each major group in this study was further divided as aged $\mathrm{RBC}$ of aged group treated with L-carnosine (AcaA), young RBC of aged group treated with L-carnosine (AcaY); aged RBC of young group treated with L-carnosine (YcaA), young RBC of young group treated with L-carnosine (YcaY), aged RBC of aged saline group (AsA), young RBC of aged saline group (AsY); aged RBC of young saline group (YsA), young RBC of young saline group (YsY). Then, RBC deformability of each group was measured.

\section{RBC deformability measurements}

Ektacytometry for testing red blood cell deformability needs about 5-6 microliter of blood or blood cell suspension for a test. RBC deformability (the ability of the entire cell to adopt a new configuration when subjected to applied mechanical forces) was determined by laser diffraction analysis using an ektacytometer (LORCA, RR Mechatronics; Hoorn, The Netherlands). The system has been described elsewhere in detail (10). Briefly, a low Hct suspension of RBC in 4\% polyvinylpyrrolidone 360 solution (MW 360 kD, Sigma P 5288, St. Louis, MO) was sheared in a Couette system composed of a glass cup and a precisely fitting bob. A laser beam was directed through the sheared sample, and the diffraction pattern produced by the deformed cells was analyzed by a microcomputer. On the basis of the geometry of the elliptical diffraction pattern, an elongation index (EI) was calculated for 9 shear rates between 0.3 and 30 Pascal $(\mathrm{Pa})$ as: $\mathrm{EI}=(\mathrm{L}-\mathrm{W}) /(\mathrm{L}+\mathrm{W})$, where $\mathrm{L}$ and $\mathrm{W}$ are the length and width of the diffraction pattern, respectively. An increased EI at a given shear stress indicates greater cell deformation and hence greater RBC deformability. All measurements were carried out at $37^{\circ} \mathrm{C}$.

\section{Measurements of RBC aggregation}

RBC aggregation was also determined by LORCA as described elsewhere (11). The aggregation measurement in that LORCA system requires about 1 milliliter of blood sample. The measurement is based on the detection of laser back-scattering from the sheared (disaggregated), then unsheared (aggregating) blood, performed in a computer-assisted system at $37^{\circ} \mathrm{C}$. Back-scattering data were evaluated by the computer and the aggregation index (AI), aggregation half-time $\left(t_{1 / 2}\right)$, which shows the kinetics of aggregation, and the amplitude (AMP), which is a measure for the total extent of aggregation, were calculated based on the fact that there is less light back-scattered from aggregating red cells. The hematocrit (Hct) of the samples used for aggregation measurements was adjusted to $40 \%$ and blood was fully oxygenated.

\section{Measurement of plasma total oxidant status}

The total oxidant status (TOS) of plasma was measured using a novel automated colorimetric measurement method for TOS developed by Erel (7). In this method, oxidants present in the sample oxidize the ferrous ion-Odianisidine complex to ferric ion. The oxidation reaction is enhanced by glycerol molecules, which are abundantly present in the reaction medium. The ferric ion makes a colored complex 


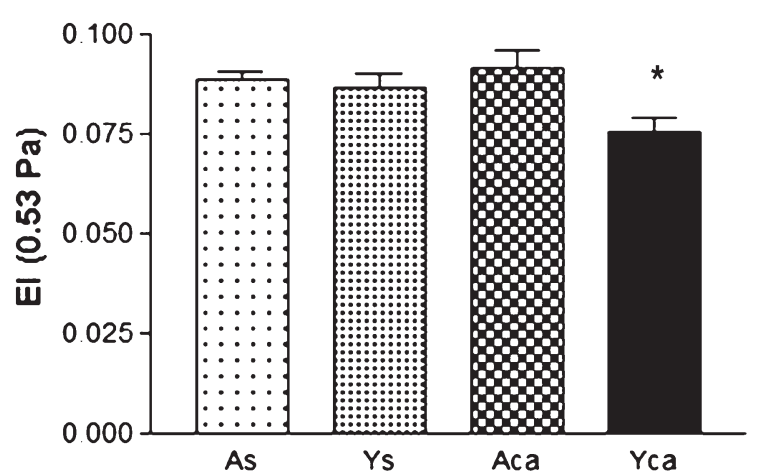

Fig. 1: Non-fractionated RBC Elongation Index (EI) of aged and young rats measured at a shear stress of $0.53 \mathrm{~Pa}$. Data presented as mean \pm standard error, *: Difference from Aca, $\mathrm{p}<0.05$. As: Aged saline group, Ys: Young saline group, Aca: Aged group treated with L-carnosine, Yca: Young group treated with L-carnosine

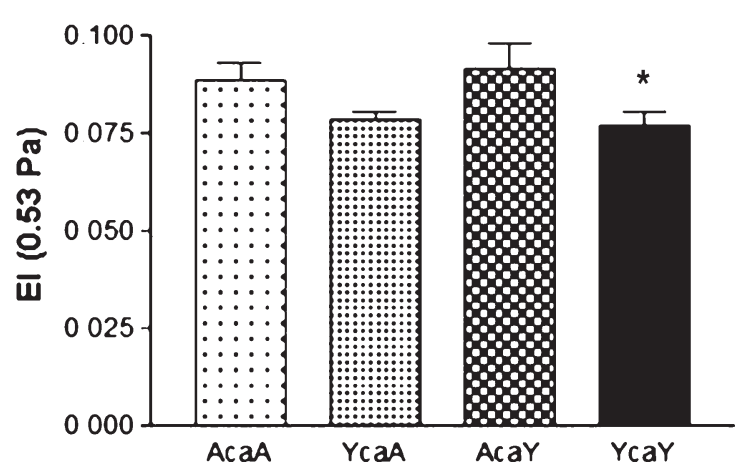

Fig. 3: Elongation Index (EI) of L-carnosine groups measured at a shear stress of $0.53 \mathrm{~Pa}$. Data presented as mean \pm standard error, *: Difference from AcaY, p < 0.05. AcaA: Aged RBC of aged group treated with L-carnosine, YcaA: Aged RBC of young group treated with L-carnosine, AcaY: Young RBC of aged group treated with L-carnosine; YcaY:Young RBC of young group treated with L-carnosine

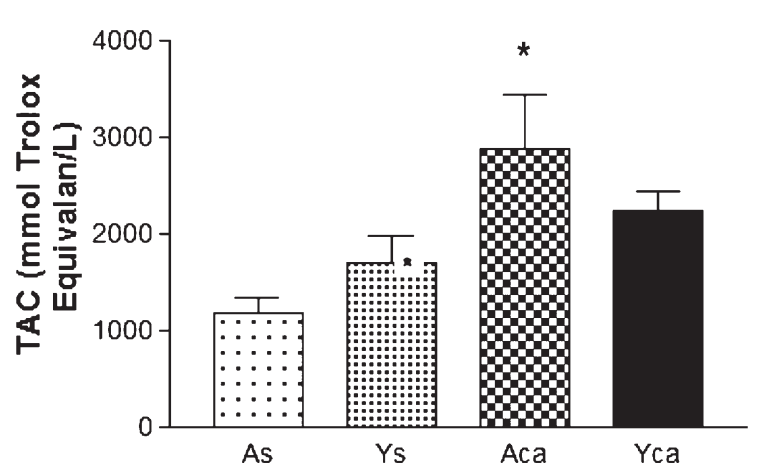

Fig. 5: Plasma Total Antioxidant Capacity (TAC) of aged and young rats. Data presented as mean \pm standard error, *: Difference from the As group, $\mathrm{p}<0.05$. As: Aged saline group, Ys: Young saline group, Aca: Aged group treated with L-carnosine, Yca: Young group treated with L-carnosine

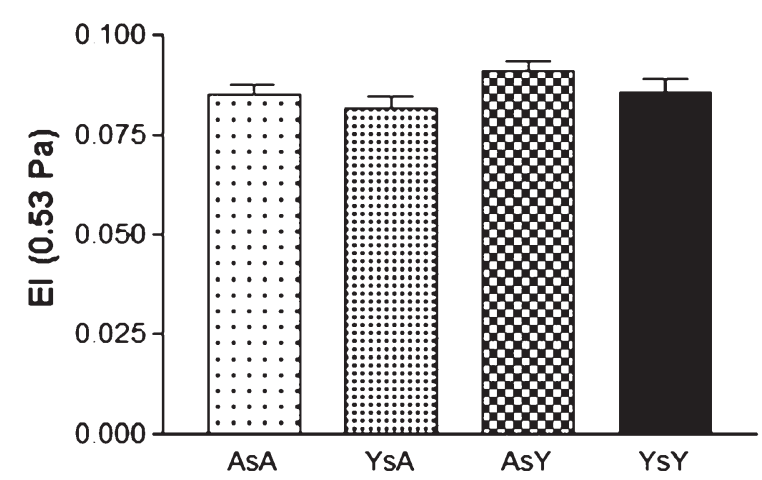

Fig. 2: Elongation Index (EI) of saline groups measured at a shear stress of $0.53 \mathrm{~Pa}$. Data presented as mean \pm standard error. AsA: Aged RBC of aged saline group, YsA: Aged RBC of young saline group, AsY: Young RBC of aged saline group, YsY: Young RBC of young saline group

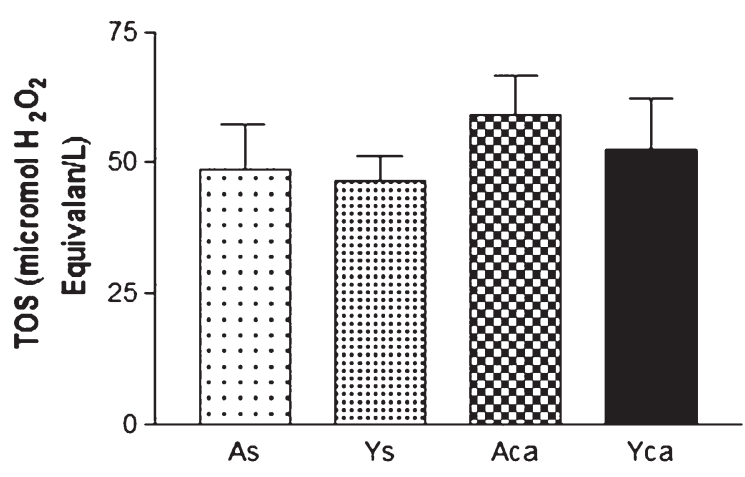

Fig. 4: Plasma Total Oxidant Status (TOS) of aged and young rats. Data presented as mean \pm standard error. As: Aged saline group, Ys: Young saline group, Aca: Aged group treated with L-carnosine, Yca: Young group treated with L-carnosine

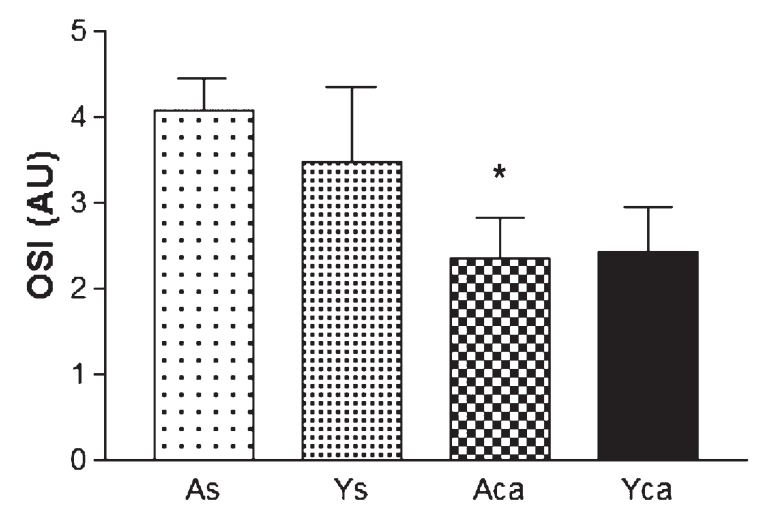

Fig. 6: Plasma Oxidative stress index (OSI) of aged and young rats. Data presented as mean \pm standard error, *: Difference from the As group, $\mathrm{p}<0.05$. As: Aged saline group, Ys: Young saline group, Aca: Aged group treated with L-carnosine, Yca: Young group treated with L-carnosine 
with xylenol orange in an acidic medium. The color intensity, which can be measured spectrophotometrically, is related to the total amount of oxidant molecules (lipids, proteins) present in the sample. The assay is calibrated with hydrogen peroxide, and the results are expressed in terms of micromolar hydrogen peroxide equivalent per liter ( $\mu \mathrm{mol} \mathrm{H}_{2} \mathrm{O}_{2}$ equiv/L).

\section{Measurement of plasma total antioxidant capacity}

The total antioxidant capacity (TAC) of plasma was measured using a novel automated colorimetric measurement method for TAC developed by Erel (6). In this method the hydroxyl radical, the most potent biological radical, is produced by the Fenton reaction and reacts with the colorless substrate O-dianisidine to produce the dianisyl radical, which is bright yellowish-brown in color. Upon the addition of a plasma sample, the oxidative reactions initiated by the hydroxyl radicals present in the reaction mix are suppressed by the antioxidant components of the plasma, preventing the color change and thereby providing an effective measure of the TAC of the plasma. The assay results are expressed as mmol Trolox equiv/L.

\section{Calculation of oxidative stress index}

The ratio of TOS to TAC is referred as the oxidative stress index (OSI). The OSI is calculated according to the following formula: OSI (arbitrary unit) $=\left(\mathrm{TOS}\left(\mu \mathrm{mol} \mathrm{H}_{2} \mathrm{O}_{2}\right.\right.$ equiv/L)/TAC (mmol Trolox equiv/L) $) \times 100(5)$.

\section{Statistical analysis}

Descriptive statistics are given as mean \pm standard error (SE). To compare diferences between four independent groups, the Kruskal Wallis Variance Analysis Test was used, and then to compare sub groups, the Mann Whitney U Test with Bonferroni Corecction was used. Statistical significance was set at $\mathrm{P}<0.05$. The statistical analyses were performed with the statistical package program SPSS version 10.0 .

\section{Results}

RBC deformability (assessed as the elongation index, $\mathrm{EI}$ ) for the RBCs of all experimental groups was measured at 9 shear stresses between 0.3 and $30 \mathrm{~Pa}$ and EI values measured at $0.53 \mathrm{~Pa}$ are presented. This pattern of altered RBC deformability was similar for the other shear stresses. $0.53 \mathrm{~Pa}$ was used as the differences between groups is most prominent in the shear stress. Figure 1 demonstrates non-fractionated RBC deformability (i.e. the elongation index EI) of aged and young rats as well as the effect of L-carnosine treatment. RBC deformability of young rats treated with L-carnosine (Yca) was significantly lower than aged rats treated with L-carnosine (Aca) $(p<0.05)$.
RBC deformability results of young and aged RBC of young saline groups (YsY and YsA) and aged saline groups (AsY and AsA) are presented in Figure 2. No significant alterations in RBC deformability were observed between the groups. Figure 3 demonstrates RBC deformability results of density separated $\mathrm{RBC}$ of aged and young L-carnosine groups. EI of young $\mathrm{RBC}$ of young group treated with L-carnosine (YcaY) was significantly lower than young $\mathrm{RBC}$ of aged group treated with L-carnosine (AcaY) $(\mathrm{p}<0.05)$.

The aggregation index (AI), aggregation half time $\left(\mathrm{t}_{1 / 2}\right)$ and aggregation amplitude (Amp) of non-fractionated RBC of aged and young rats as well as the effect of L-carnosine treatment are shown that in Table 1. No significant alterations in aggregation parameters were observed between the groups.

Tab. 1: Aggregation parameters of young, aged rats treated with L-carnosine or saline

\begin{tabular}{|l|c|c|c|}
\hline & AI $(\%)$ & $\mathrm{t}_{1 / 2}(\mathrm{~s})$ & Amp (Au) \\
\hline As & $67.86 \pm 2.72$ & $1.65 \pm 0.26$ & $20.94 \pm 1.10$ \\
\hline Ys & $55.95 \pm 4.31$ & $3.32 \pm 0.63$ & $18.35 \pm 1.29$ \\
\hline Aca & $71.31 \pm 2.94$ & $1.37 \pm 0.26$ & $22.21 \pm 1.71$ \\
\hline Yca & $59.74 \pm 2.74$ & $2.51 \pm 0.36$ & $23.50 \pm 2.12$ \\
\hline
\end{tabular}

$\mathrm{AI}$, aggregation index; $\mathrm{t}_{1 / 2}$, aggregation half time; Amp, amplitude of aggregation. Descriptive statistics are given as mean $\pm \mathrm{SE} ; \mathrm{n}=7$

L-carnosine treatment had no statistically significant effect on the TOS level of aged and young rats (Figure 4). Plasma TAC levels were significantly higher in the L-carnosine-treated aged group compared to aged control group $($ As, $\mathrm{p}<0.05)$ (Figure 5). On the other hand, although L-carnosine treatment caused decrements in plasma OSI, the result was statistically significant only for the L-carnosine-treated aged group (Aca) compared to aged control group (As, p < 0.05) (Figure 6).

\section{Discussion}

Flow, deformability and aggregability of blood cells are main components of hemorheology. In microcirculation, where cells must deform to pass through narrow capillaries, deformability and aggregation of RBCs are the major determinants of resistance to flow. The ability of the entire $\mathrm{RBC}$ to deform is of crucial importance for performing its function of oxygen delivery and it is also a determinant of the cell survival time in the circulation (4). In this study, the effects of L-carnosine treatment on hemorheological parameters during biological aging and cellular aging of erythrocytes in rats were demonstrated. The results of the current study indicate that, L-carnosine has a negative effect on $\mathrm{RBC}$ deformability in young rats compared to aged rats and anti-oxidant activity of L-carnosine in aged rats is more 
prominent than young rats. Results of some previous studies have shown that RBC deformability decreased during biological aging $(1,26)$. One of these studies, performed in our laboratory demonstrated that RBC deformability of aged Wistar albino rats (18 months) was significantly lower than young rats (15). In contrary with these results, Tugral et al., have shown that RBC deformability of aged individuals was higher than young ones. However, this increase was not statistically significant (27).

Previously conducted studies reported that lifetime of RBC shortens with the aging of the individual and as a result of this process increased hematopoiesis causes enhancement of the number of young RBCs in the circulation $(16,19)$. In the current study, RBC deformability of young and old control Sprague Dawley rats were not found to be different from each other (Figure 1). Although not measured, the possible increment of young erythrocytes in the blood of rats at later periods of old age ( 24 months) used in the our study may be an explanation for this issue.

The effect of in vivo L-carnosine administration $(250 \mathrm{mg} / \mathrm{kg})$ on RBC deformability of young and aged rats was also determined in the current study. Aydogan et al. (1) have previously demonstated that, L-carnosine improves RBC deformability impaired with $\mathrm{H}_{2} \mathrm{O}_{2}$ administration and the improvement was more prominent in young rats (3 months) compared to old rats (12 months). Although L-carnosine at the dose and duration injected in the current study induced a slight increment in the RBC deformability of aged rats, it did not cause a significant alteration in the deformability of the non-fractioned RBCs of the young and aged rats in comparison with their respective controls (Figure 1). On the other hand, in contrary with the results of Aydogan et al., RBC deformability of young rats after L-carnosine treatment was lower than that of aged rats and the difference was statistically significant. As far as we know, our study is the first one in the literature examining in vivo effects of L-carnosine on hemorheological parameters in rats. The difference between the findings of our study and those of Aydogan et al. can be due to the fact that, they conducted an in vitro experiment whereas our study is an in vivo one.

We also compared the deformability of young and aged RBCs of young and aged control rats (Figure 2). Previous studies showed that as the duration where RBCs remain in the circulation increases, $\mathrm{RBC}$ deformability decreases $(2,22,25)$. Waugh et al. suggested that while RBC surface area decreases as the RBCs age, intracellular viscosity increases in consequence of cellular dehydration and these changes can explain the decrease in RBC deformability in line with cellular aging (29). However, it was reported in another study that the shear elastic modulus, which is one of the primary determinants of the elastic behavior of cells, does not change at a significant level in relation with in vivo RBC aging. Additionally, the cell surface-volume ratio of aged RBCs is not very different from that of young $\mathrm{RBCs}$ enough to prevent the circulation of $\mathrm{RBCs}$ in microcirculation (18). The results of our study also demonstrate that although the alteration was not at a significant level, RBC deformability decreases as the RBC's age increases in both young and old rats (Figure 2). This finding is in concordance with the literature $(2,25,29)$.

Deformability of density separated RBCs after L-carnosine injection was also determined in the current study (Figure 3). It was observed that L-carnosine injection decreases deformability of young RBCs (YcaY) of young rats in a statistically significant level when compared with the deformability of the young RBCs of aged rats (AcaY). When the deformability of aged RBCs of aged rats (AcaA) after L-carnosine treatment was compared with aged erythrocytes of young rats (AcaY) a tendency to decrease was observed, but it was not at a statistically significant level. These results are in concordance with the decrease of the non-fractioned RBCs of young rats in Figure 1, observed after L-carnosine injection. At the dose and application duration used herein, L-carnosine causes decrement in the deformability of young rats, and their young RBCs, due to an unknown mechanism. On the other hand, the -relative increment- in the deformability of young and aged RBCs of aged rats following L-carnosine treatment, observed in both Figure 1 and 3, can serve as an advantageous compensatory factor to prevent circulation disorders that may occur as a result of ageing of the organism and RBC. Considering these data, it can be speculated that unlike young rats, L-carnosine treatment can contribute the improvement of circulation in aged rats.

Another hemorheological parameter determined in the current study is the RBC aggregation which is the reversible adhesion of adjacent erythrocytes (22). While the amplitude af aggregation (AMP), is a static parameter providing information about the magnitude of the total RBC aggregation, aggregation half-time $\left(\mathrm{t}_{1 / 2}\right)$ is a kinetic parameter indicating the rate of the aggregation. Aggregation index (AI), on the other hand, can be considered as a function of the static and kinetic characteristics of RBC aggregation (11). Despite the fact that the literature involves studies reporting increment of RBC aggregation in line with ageing $(14,28)$, to our knowledge our study is the first one that examining the effects of L-carnosine on RBC aggregation at old age. Although the alterations were not statistically significant, the results of our study indicating increment of RBC aggregation during againg are in concordance with the literature. Similarly, no statistically significant effect of L-carnosine could be found on the examined RBC aggregation parameters (Table 1).

Within the scope of our study, TOS was measured as an indicator of the oxidative stress in the blood plasma of young and aged rats (6), and TAC measurement was carried out in order to test the antioxidant capacity of the organism (20). Furthermore OSI, that reflects the redox balance between oxidation and antioxidation, was measured as an indicator of oxidative stress $(5,7,20)$. Throughout their lifetime, RBCs are exposed to various physical and 
chemical stresses. One of these is the oxygen radicals that have a reactive nature and that can harm cellular structures (9). It was reported that the oxidant damage caused by the oxygen radicals is also effective on RBC deformability and decreases RBC deformability $(4,23)$. L-carnosine is a dipeptide known to have antioxidant activity. The results of our study have shown that L-carnosine injection to aged rats increases antioxidant defense and significantly lowers oxidative stress index compared to aged control rats (Figure 5 and Figure 6). This finding is consistent with the literature. The finding that L-carnosine exhibits its antioxidant activity particularly in aged rats may be related to the improved RBC deformability observed in aged rats following L-carnosine injection (Figure 1). As it is known, an increase in $\mathrm{RBC}$ deformability can positively affect tissue oxygenation by facilitating the passage through capillary circulation. One of the aging theories is related with the oxidant damage increasing in line with ageing (30). The results of this study emphasize the usefulness of L-carnosine therapy in aged animals with the purpose of regulating circulation and protecting aged individuals against oxidant damage. Further mechanistic studies will be necessary to understand the observed changes in hemorheological parameters in RBC subpopulation and the mechanisms of these alterations as well.

\section{References}

1. Aydogan S, Yapislar H, Artis S, Aydogan B. Impaired erythrocytes deformability in $\mathrm{H}_{2} \mathrm{O}_{2}$-induced oxidative stress: protective effect of L-carnosine. Clin Hemorheol Microcirc 2008; 39(1-4): 93-98.

2. Bor-Kucukatay M, Meiselman HJ, Bașkurt OK. Modulation of density-fractionated RBC deformability by nitric oxide. Clin Hemorheol Microcirc 2005; 33(4): 363-67.

3. Chien S. The red blood cell: biophysical behavior of red cells in suspensions. In Surgenor DM, ed. The red blood cell. New York: Academic Press, 1975: 1031.

4. Chien S. Red Cell Deformability and its Relevance to Blood Flow. Annual Review of Physiology 1987; 49: 177-92.

5. Demirbag R, Gur M, Yilmaz R, Kunt AS, Erel O, Andac MH. Influence of oxidative stress on the development of collateral circulation in total coronary occlusions. Int J Cardiol 2007; 116: 14-19.

6. Erel O. A novel automated method to measure total antioxidant response against potent free radical reactions. Clin Biochem 2004; 37: 112-19.

7. Erel O. A new automated colorimetric method for measuring total oxidant status. Clin Biochem 2005; 38: 1103-11.
8. Guiotto A, Calderan A, Ruzza P, Borin G. L-carnosine and L-carnosine-related antioxidants: A review. Curr Med Chem 2005; 12(20): 2293-315.

9. Gutteridge JM, Halliwell B. Free radicals and antioxidants in the year 2000 A historical look to the future. Ann NY Acad Sci 2000; 899: 136-47.

10. Hardeman MR, Goedhart PT, Dobbe JGG, Lettinga KP. Laser-assisted optical rotational cell analyzer (LORCA). 1. A new instrument for measurement of various structural hemorheological parameters. Clin Hemorheol 1994; 14: 605-18.

11. Hardeman MR, Dobbe JG, Ince C. The Laser-assisted Optical Rotational Cell Analyzer (LORCA) as red blood cell aggregometer. Clin Hemorheol Microcirc 2001; 25: 1-11.

12. Hipkiss AR. L-carnosine, a protective, anti-ageing peptide? Int J Biochem Cell Biol 1998; 30(8): 863-68.

13. Hipkiss AR, Brownson C. A possible new role for the anti-ageing peptide L-carnosine. Cell Mol Life Sci 2000; 57(5): 747-53.

14. Jayavanth S, Singh M. Changes in erythrocyte aggregation and deformability during human ageing. Current Science 2002; 82: 191-96.

15. Kucukatay V, Bor-Kucukatay M, Kocamaz E, Erken G. Effect of Sulfite Treatment on Erythrocyte Deformability in Young and Aged Rats. Toxicol Mech Methods 2009; 19(1): 19-23.

16. Magnani M, Rossi L, Stocchi V, Cucchiarini L, Piacentini G, Fornaini G. Effect of age on some properties of mice erythrocytes. Mech Ageing Dev 1988; 42(1): $37-47$.

17. Mohandas N, Chasis JA, Shohet SB. The influence of membrane skeleton on red cell deformability, membrane material properties and shape. Semin Hematol 1983; 20: 225-42.

18. Nash GB, Meiselman HJ. Red cell and ghost viscoelasticity, effects of hemoglobin concentration and in vivo aging. Biophys J 1983; 43: 63-73

19. Pinkofsky HB. The effect of donor age on human erythrocyte density distribution. Mech Ageing Dev 1997; 97(1): 73-79.

20. Rabus M, Demirbağ R, Sezen Y et al. Plasma and tissue oxidative stress index in patients with rheumatic and degenerative heart valve disease. Turk Kardiyol Dern Ars 2008; 36(8): 536-540.

21. Sentürk UK, Gündüz F, Kuru O et al. Exercise-induced oxidative stress leads hemolysis in sedentary but not trained humans. J Appl Physiol 2005; 99(4): 1434-41.

22. Shiga T, Maeda N, Kon K. Erythrocyte rheology. Crit Rev Oncol Hematol 1990; 10(1): $9-48$.

23. Srour MA, Bilto YY, Juma M, Irhimeh MR. Exposure of human erythrocytes to oxygen radicals causes loss of deformability, increased osmotic fragility, lipid peroxidation and protein degradation. Clin Hemorheol Microcirc 2000; 23(1): $13-21$.

24. Stuart J, Nash GB. Red cell deformability and hematological disorders. Blood Rev 1990; 4: 141-147

25. Tillmann W, Levin C, Prindull G, Schröter W. Rheological properties of young and aged human erythrocytes. Klin Wochenschrift 1980; 58: 569-74.

26. Tozzi-Ciancarelli MG, Fedele F, Tozzi E et al. Age dependent changes in human erythrocyte properties. Clin Hemorheol 1989; 9: 999-1007.

27. Tuğral E, Yalçın Ö, Başkurt OK. Effect of Donor Age on the Deformability and Aggregability of Density-Separated Red Blood Cells. Turk J Hematol 2002; 19(2): 303-308.

28. Ward KA, Baker C, Roebuck L, Wickline K, Schwartz RW. Red blood cell deformability: Effect of age and smoking. Age 1991; 14(3): 73-77.

29. Waugh RE, Narla M, Jackson CW, Mueller TJ, Suzuki T, Dale GL. Rheologic properties of senescent erythrocytes: loss of surface area and volume with red blood cell age. Blood 1992; 79(5): 1351-8.

30. Weinert BT and Timiras PS. Invited review: Theories of aging. J Appl Physiol 2003; 95: 1706-16.

Received: 28/02/2012

Accepted in revised form: 16/01/2013

\section{Corresponding author:}

Gülten Erken, Balikesir University, Faculty of Medicine, Department of Physiology, Balikesir, Turkey; e-mail: gulemmun (a) gmail.com 